\title{
OPTIC NEURITIS, PAPILLITIS, AND NEURONAL RETINOPATHY*
}

\author{
BY \\ SIMON BEHRMAN \\ London
}

THE word "neurone" in ancient Greece, in addition to signifying "nerve", also meant "a bow-string". The toughness of the optic nerve might be regarded as a vindication of this etymology. It is not uncommon to find the optic nerve reduced to a mere thread by a tumour or aneurysm at a time when vision is still intact. But the optic nerve is not, in fact, a nerve, but a tract of white matter. This, and the viability of this nerve, are preliminaries to any consideration of the optic neuropathies.

\section{The Optic Nerve}

As a tract of white matter of the central nervous system, the optic nerve is unique from the morphological point of view, in having its elements-the myelinated axons - separated into fascicles by abundant inward septa of pia mater. Within these septa are contained connective tissue elements (fibroblasts and collagen) and blood vessels. These vessels can be traced to originate from the arterial pial plexus surrounding the nerve. Collateral branches to the optic nerve from the internal carotid, ophthalmic, and central retinal arteries, and the recurrent pial branches of the circle of Zinn all contribute to the formation of the pial arterial plexus. Injection experiments indicate the very extensive nature of this anastomosis. Singh and Dass (1960a) found that these anastomoses were usually large enough to allow blood to flow from one of the contributing vessels to another.

These authors also claim to have established, in a few specimens, an anastomosis between vessels within the substance of the nerve.

In addition to the arterial pial plexus-reminiscent of the arterial vasocorona of the spinal cord - they recorded inconstant and variable branching of the central retinal artery within the optic nerve.

As seen by the electron microscope, the pia mater enveloping the optic nerve is a basement membrane which, fused with the underlying glial membrane, forms the pia-glia. Vessels which come into contact with the pia-glia have, upon entering the subarachnoid space, acquired an arachnoid cuff. Branches of these vessels with their surrounding arachnoid "tubes" form the core of the numerous pia-glia septa of the optic nerve.

\section{Optic Neuritis}

In the 19th century, during which the term was in wide use, its precise nosographic significance underwent many mutations, and its continued use may be questioned. Virologists widely agree that among the neurotropic viruses none has been identified as causing primary damage to the white matter. Infective agents are injurious to the 
optic nerve in so far as they cause inflammation of the leptomeninges. It must be recalled here that leptomeninges not only surround the nerve but by numerous folds honeycomb the substance of nerve.

Among infective agents causing meningitis are a number of viruses and many pathogenic organisms. The blood supply of the nerve, being derived from pial anastomosis indissolubly linked with leptomeninges, is thus put in jeopardy and may occasionally be severely truncated in meningitis. Such damage by ischaemia is particularly liable to ensue in the more chronic forms of leptomeningitis. When the leptomeningitis is due to endarteritis obliterans of the small meningeal vessels, as in syphilis, ischaemic damage of the nerve is especially liable to supervene. This in essence constitutes the pathological process of "interstitial syphilitic optic neuritis" and "tabetic optic atrophy" (Bruetsch, 1947). Except in a few cases in which there is early involvement of central portion of the visual fields, and consequently early recognition of the disorder, antisyphilitic treatment is usually disappointing. Infection of the posteriorly-placed accessory nasal sinuses is another, albeit rare, cause of optic neuritis.

Infiltration of leptomeninges of the optic nerve with metastatic tumour cells, either from a carcinoma or melanoma or with leukaemic cells, can lead to early unexplained loss of vision (Katz, Valsamis, and Jampel, 1961; Weizenblatt, 1959; Huber, 1961).

\section{Optic Neuropathies due to Angiopathies}

Attention has already been drawn to the inordinate number of anastomoses among the vessels contributing to the blood supply of the optic nerve. There is one between the ophthalmic artery and branches of the external carotid, one between the various collateral branches supplying the optic nerve by means of the pial plexus, and finally one between various branches of this plexus within the substance of the optic nerve. Amid such a free system of communication an isolated occlusive vascular lesion is unlikely to cause any circulatory disturbance. To bring about ischaemia there must be extensive occlusive disease of the main vessels supplying the nerve and/or of the pial plexus and its intraneural branches. Such wide involvement of vessels of different calibre is exceptional in classical arteriosclerosis. Furthermore, the condition being tardy, new compensatory anastomotic channels have time to develop in arteriosclerosis. The situation is radically different in the case of syphilitic endarteritis, as already mentioned, and a group of acute or sub-acute angiopathies such as giant cell arteritis, periarteritis nodosa, and disseminated lupus erythematosus. Amaurosis due to retrobulbar optic nerve ischaemia can occasionally be seen as an early clinical presentation of any of this group of diseases.

\section{Demyelinating Diseases}

Except for a greater proclivity to be affected, the optic nerve does not in any way differ in its response to demyelinating diseases from white matter in other parts of the central nervous system.

The morphology of the lesions exhibits a similar range of reactions, from demyelination with relatively intact axons to focal necrosis. The intensity of glial and inflammatory reactions are also subject to much variation. The tendency for the lesions to become confluent, and their symmetry on both sides of the axis, are 
equally unsatisfactory criteria for classification. The clinician, in order to introduce some order, has attempted to arrange these diseases on the basis of positive family history or recent exposure to virus infection or a tendency to relapse. As far as the optic nerve is concerned the asymmetrical, relapsing type of optic neuritis is regarded as diagnostic of multiple sclerosis, whereas cases in which both optic nerves are simultaneously involved are placed into the category of "Devic's disease" (a clinical variant of demyelinating encephalomyelitis).

Since, by analogy with the brain, the blood vessels are the only pain-sensitive structures of the optic nerve, the common symptom of optic neuritis-pain on extreme ocular movement-must originate from the pain receptors of the blood vessels of the nerve. The orbital part of the optic nerve differs from the intracranial part in having a far greater number of mesodermal partitions with their core of blood vessels. Increased mobility of this part of the nerve must account for this anatomical difference.

When these partitions show inflammatory reaction, as evidenced by cell infiltration characteristic of inflammations, a lowering of the pain threshold must occur, and this would account for pain on extreme ocular movements. This inflammatory reaction can be a striking feature both of demyelinating disease and of experimental allergic encephalo-myelitis (Symonds, 1924). Since this characteristic ocular pain often precedes the amblyopia of optic neuritis by many days, it must be concluded that the localized meningeal inflammatory reaction antedates demyelination by this period. Occasionally tenderness of the globe of the affected eye may be a notable feature. This hyperalgesia corresponds to the cutaneous tenderness of the skin of the chest over the left side following attacks of angina pectoris (Sturge, 1883). A similar tenderness of the eyeball can be produced experimentally by noxious stimulation of remote structures within the trigeminal area (Wolff, 1948).

As a rule, in demyelinating diseases, visual failure is of sudden onset. Very occasionally, however, in otherwise classical cases of multiple sclerosis, the typical monocular central scotoma is small at the onset and then very slowly expands in all directions.

\section{Papillitis}

Papillitis should, of course, signify a morbid condition in which the optic nervehead is the seat of inflammation. Used in this sense, this terminology is inappropriate in demyelinating diseases, since the site of the inflammatory process must be retrobulbar, as myelination ceases a short distance behind the lamina cribrosa. Yet swelling of the disc can be very considerable in these diseases and can mimic ophthalmoscopically severe papilloedema in every respect. Furthermore, these changes at the disc are capable of such rapid resolution as to render an inflammatory process unlikely. It must be concluded, therefore, that the swelling of the nervehead is due to oedema as the ophthalmoscopic appearance suggests. Thus, the inflammatory process within the nerve seems, under certain conditions, to engender oedema of the nervehead. Concerning one such condition there is a wide measure of agreement, viz. for swelling of the disc to occur the plaque of demyelination must be situated in the most anterior segment of the medullated part of the optic nerve. Clearly the topography of the lesion is of paramount importance, and its significance becomes 
manifest on consideration of certain established morphological facts, of the pressure relationships prevailing in the terminal portion of the optic nerves, and also of some experimental observations.

Morphological Considerations.-The perivascular or Virchow-Robin space, which surrounds the arteries and veins within the central nervous system down to their finest branches, is a structure peculiar to the central nervous system. That this space is not an artefact is established by the manner in which, under pathological conditions, lymphocytes are entrapped within it.

There is some uncertainty whether such perivascular spaces also surround capillaries. In specimens which Krückmann secured immediately after an execution, he claims to have demonstrated such pericapillary spaces in the retina (Krückmann, 1917). He described outer glial and inner pial layers "stuck together like sheets of paper"-jointly forming a mantle enclosing the pericapillary "lymph space". This twin-layered tube clearly corresponds to the Pialmembran described by Schaltenbrand and Bailey (1928) as enveloping the capillaries in the central nervous system.

Some confirmation of the existence of pericapillary spaces in the central nervous system has been provided by electron microscopy (van Breeman and Clemente, 1955). Having set himself the task of demonstrating histologically the continuity between the pericapillary and perivascular spaces in the retina, Krückmann was obliged to admit that he had been unsuccessful, but he still expressed the belief that such continuity did in fact exist.

The perivascular space surrounding the central retinal artery within the optic nerve is so extensive as to be demonstrable macroscopically; within the perineural subarachnoid space, the perivascular space surrounding the central retinal artery is contained within an "arachnoid tube" (Singh and Dass, 1960b). Behr (1935) repeatedly emphasized the absence of any demonstrable union between the adventitia of the central retinal artery and the dura where the artery traverses obliquely the dural sheath. This observation would justify the inference that the perivascular space extends also to the intradural segment of the artery.

Barological Considerations.-Of the perivascular spaces Greenfield said that both in constitution and function they must be considered as "lymphatic channels in C.N.S." (Greenfield, 1958). While this concept could be justly extended to include the perivascular spaces of the retina and optic nerve, the image used by Woollam and Millen (1954) of "perivascular spaces as backwaters in which there is a gentle eddy in both directions" cannot be applied to the eye, because of the known pressure gradient between the intra-ocular and retrobulbar parts of this system. A constant flow in a retrobulbar direction must therefore be assumed in these channels.

Experimental Observations.-Some 80 years ago Gifford (1886) injected particulate matter into the vitreous of albino rabbits and reported his observations based on some forty experiments as follows:

\footnotetext{
"The results obtained were definite and constant. When a small drop of sterilized water containing india-ink or cinnabar in suspension is injected into the vitreous, there is generally little or no reaction, and on the next day or later, according as the injection was made further backwards or
} 
forward, one sees with the ophthalmoscope the particles collecting in the optic excavation, and if the animal is killed a day or two after this the macroscopic examination of longitudinal sections of the optic nerve show the whole course of the central canal (of the nerve) marked by a black (or if cinnabar is used, red) line which passes out of the nerve with the vessels, and can be traced, with the naked eye, for a short distance toward the rear of the orbit. Microscopically, one sees that granules are contained in the lymph-spaces around vessels ... to all appearances free in long lines parallel to the vessels... Granules are found, especially, close to the point where the main vessels pass through the pial sheath toward the orbit.... Outside the optic nerve the pigment keeps close to the sheath of the vessels and has been traced to the posterior end of the orbit".

This experimental demonstration of a retino-orbital flow in the rabbit may well have been facilitated by the absence of the lamina cribrosa in this animal (Tansley, 1956). Though inconclusive - in view of the nature of the experiment - the observations by Berens and Posner on the living human eye revealed a similar migration of injected material into the intraneural perivascular space (Berens and Posner, 1933).

The Retino-orbital Flow.-The morphological evidence serves to establish the basis for the existence of a channel between the retina and orbit, the barological arguments help to determine the direction of tissue-fluid flow, and Gifford's experiments provide some experimental confirmation for the existence of an outflow into the orbital space. Some degree of retinal oedema might be expected if this tissue-fluid current were interrupted. However, the arrangement of the radial fibres of Mueller impart such a degree of compactness to the nerve fibre layer of the retina as to ensure that oedema is circumscribed to the relatively loose tissue of the nervehead (Wolff, 1951). The laxity here is due not only to the absence of the fibres of Mueller but also to the attenuation of the astrocytes at the papilla where they become "spider cells", without attachment to capillary vessels (Wolter, 1957). Swelling of white matter in and around the plaque of demyelination is evidently capable of blocking temporarily the retino-orbital flow.

There must be many diverse pathological processes (e.g. ischaemia, neoplasia, sarcoidosis) which, by involving the most anterior portion of the optic nerve and effecting a similar blockage-perhaps only temporary-of the perivascular retino-orbital channel, can engender the syndrome of indeterminate "papillitis". This concept of oedema of the nervehead, in consequence of a variety of retrobulbar lesions of the optic nerves, is in no way at variance with a "true" papillitis of inflammatory nature, occurring in association with inflammatory affections of the uveal tract.

\section{"Senile Papillitis"}

Particular reference must now be made to a small group of cases of "papillitis" showing a number of congruous features which may indicate that, from the point of view of nosography, these cases can be grouped together:

(1) Senescence of the patients.

(2) Monocular amblyopia with swelling of the corresponding optic disc, and, occasionally homolateral headache of moderate severity at onset.

(3) Similar involvement of the other eye after an interval of 6 to 12 months.

(4) After vision has become monocular (as a result of loss of vision of one eye) conditions obtain which allow accurate observation of the manner in which failure of vision develops 
in the second eye. When thus determined, the amblyopia is found not to be of apoplectic onset but to advance slowly.

(5) Erythrocyte sedimentation rate is within normal limits.

A case which presented all these features was described by Rintelen (1961).

A woman aged 53 years was found to have a swollen optic disc when examined on account of rapid decline of vision to $6 / 60$. 3 months later, vision of the other eye became obscured, eye movements were painful, and again there was swelling of the affected optic disc. "Vision continued to deteriorate daily".

Whereas there was no improvement of vision in the first eye affected, full recovery occurred in the second eye within 2 months.

In three similar, personally observed cases, loss of vision in the second eye also developed in an equally tardy manner after one year. Excepting for the advanced age, averaging well over 70 , the clinical features were those of papillitis due to demyelinating disease.

Rintelen presented his case under the title of "apoplexia papillae" and attributed this to "localized arteriosclerosis". Several authors have described similar cases under a variety of names while also postulating a localized sclerotic angiopathy (Kurz, 1948; François, Verriest, Neetens, and de Rouck, 1961). In view of the uncertainty of the underlying pathology it might be better to term the condition provisionally "senile papillopathy" or "senile papillitis".

Available evidence would suggest that a degree of recovery of vision is possible in some cases, and that the vision of the second eye may be spared. In a nosographically similar group of twenty cases reported by François and others (1961) under the name of "pseudo-papillites vasculaires", the second eye was involved in four cases after intervals of up to one year's duration. The absence of arteralgias and of associated constitutional symptoms, and an erythrocyte sedimentation rate within normal limits, assist to distinguish this condition from giant cell arteritis. When conditions of monocular vision obtain, the gradual loss of vision is in striking contrast with the almost apoplectic amaurosis due to giant cell arteritis. The syndrome of "optic neuritis following cataract extraction" described by Reese and Carroll (1958) bears a very close resemblance to that of "senile papillitis".

\section{Retrobulbar Neuritis}

There remains a group of amblyopias which in the clinical classifications are usually listed-by ophthalmologists and neurologists alike-under the heading of retrobulbar neuritis. These amblyopias are often bilateral and on perimetry exhibit either central or paracentral defects or an overall depression of the entire field. Except for some degree of optic atrophy ophthalmoscopy is negative when the condition is of long standing. In a few such cases in which microscopic examination of optic nerves was reported, it revealed destruction of the myelin sheaths and axis cylinders, usually maximal in central locations. The number of such cases in which retinal examination was also performed is very small. Extensive degeneration of the ganglion cell layer of the retina was found in some, as, for instance, in cases of familial optic atrophy with cerebellar ataxia (Woodworth, Beckett, and Netsky, 1959) and in a case of Leber's optic atrophy (Kwittken and Berest, 1958). 
In some, aetiological factors, such as exposure to toxic substances or dietetic deficiencies, are obvious: in others, the existence of a causative genetic factor is equally unequivocal. In many of these cases, however, the cause remains abstruse. With but a few exceptions, there is no evidence of any other involvement of the central nervous system.

In the brief survey just given of optic neuropathies it was stressed that, as regards inflammatory, neoplastic, and demyelinating reactions, there is a remarkable degree of agreement between the behaviour of the white matter of the optic nerve and that of the brain and spinal cord. If this group of amblyopias were indeed due to retrobulbar neuritis, it would be difficult to account for the consistency with which white matter elsewhere in central nervous system remains unaffected. On the other hand, if the destruction of elements of the optic nerve is not to be regarded as the primary lesion, then it follows that the nerve fibre degeneration is transynaptic, consequent upon damage of the neuronal elements of the retina.

\section{Neuronal Elements of the Retina}

The obvious anatomical homology of the outer and inner nuclear layers and the ganglion layer of the retina to the three layers of the archipallium does not require comment. It does not come as a surprise, therefore, that in some diseases, as in lipoid storage diseases for instance, there is a close correspondence in the reactions between the neurones of the retina and of the cerebral cortex.

In a recent case of carbon dioxide poisoning, there was similarly a correspondence between convolutional atrophy of the cerebrum and the ganglion cells and atrophy of the nuclear layers of the retina.

The retinal neurones show, however, a high degree of functional and structural differentiation, and this would account for some distinctive metabolic characteristics which these cells exhibit. For instance, they are even more easily damaged by hypoglycaemia than are neurones of the cerebral cortex (Papst, 1955). Furthermore, the neurones of each different layer of the retina show a high degree of metabolic specificity. Iodoacetate has a cytocidal effect on the outer nuclear layer, whereas the cells of the inner nuclear layer and of the ganglion layer can be relatively easily destroyed by the administration of sodium 1-glutamate to suckling mice (Lucas and Newhouse, 1957; Noell, 1958). Another indication of the wide metabolic differences between the neurones of the retina and those of the cortex is provided by the absence of any involvement of the retinal neurones in the vast majority of diseases known to damage the cerebral cortex. Here, therefore, there is none of the affinity which exists between the optic nerve and the white matter of the brain.

\section{Neuronal Retinopathy}

The highly differentiated retinal neurones with their idiosyncratic biochemical systems are far more likely to show disturbance of function than the relatively stable organization of the optic nerve which in its reactions duplicates closely those of white matter elsewhere. The resources of electro-physiologists are as yet inadequate to reveal-except in rather advanced cases - any dysfunction of these cells in disease (Potts, Modrell, and Kingsbury, 1960). The clinician also is able to recognize only the more severe manifestations of their disordered function. First, there are the 
cases with a clear family history of amblyopia or of one of many heredo-familial non-demyelinating diseases of the central nervous system with or without amblyopia. Leber's disease is an example of the first, and the Charcot-Marie-Tooth type of muscular atrophy of the other (Kwittken and Berest, 1958; Hoyt, 1960). Secondly, there is the group in which amblyopia can be imputed to exposure to a toxic agent, of which tobacco can be cited as the commonest example. Methylated spirit addiction as a cause of slowly progressive amblyopia, with central scotomata and optic atrophy, may also be mentioned here as it is liable to be overlooked (Morgan, 1952). Finally, there is a group in which the precise causation remains uncertain.

Here could be instanced a form of amblyopia frequently encountered in Great Britain among immigrants from Jamaica, the Lesser Antilles, Trinidad, and British Guiana (Behrman, 1962). Bilateral impairment of vision develops rapidly, either in adolescence or in early adult life, and usually no appreciable deterioration of vision occurs in subsequent years. Some degree of optic atrophy is seen in most cases. Perimetry reveals either scotomata or, rarely, centro-caecal scotomata. Defective vision is often characterized by an overall depression with appreciable peripheral field loss. Though these defects are usually symmetrical, in some cases the vision of one eye is much worse than that of the other. Noteworthy is the absence of a positive family history or evidence of malnutrition. Not only do these immigrants originate from territories as far as 1,500 miles apart, but a similar type of amblyopia is encountered among Africans from the African continent, as is illustrated by the following case:

A healthy Nigerian woman aged 22 years, complained of bilateral amblyopia which had started abruptly 5 years earlier during her last term at boarding school. Extensive laboratory investigations, including examination of cerebrospinal fluid, revealed no abnormality except minimal enlargement of the spleen and the presence of hookworm ova in the stools. The visual acuity was 6/36 in the right eye and 3/60 in the left. Satisfactory perimetry revealed extensive centro-caecal scotomata in each field of vision. No other member of her family was known to be similarly affected.

Dansey-Browning (1952), in his study of ophthalmic disease in Hong Kong, where malnutrition is prevalent, makes no mention of a similar form of amblyopia. The available evidence would thus provide some support for the hypothesis that this is an African race-influenced disorder (Behrman, 1962).

\section{Summary}

As a tract of white matter, the optic nerve is unique in having its substance intersected by numerous pial septa containing blood vessels. While resistant to known virus infections, the optic nerve, because of the deep penetration of pial septa, is liable to be involved in meningitis, both acute and chronic. Syphilitic meningitis is particularly prone to engender a form of optic neuritis. Invasion of leptomeninges by metastatic tumour cells and by leukaemic cells, if occurring in close relation to the optic nerve, can also cause optic neuropathy.

Various injection techniques reveal an extremely rich system of anastomoses among arteries contributing to the supply of the optic nerve, and also among arteries within its substance. This exuberant anastomosis confers a high degree of protection against the effects of localized obstruction of the circulation. Diffuse inflammatory angiopathies, however, are liable to engender ischaemic optic neuropathy. 
In regard to neoplasia and demyelination, white matter of the optic nerve, the brain, and the spinal cord shows a remarkable uniformity of reactions.

The ophthalmoscopic appearance and rapid resolution of the swelling of the optic disc in demyelinating optic neuritis indicate that the disc swelling is due to oedema. Morphological and barological facts and experimental observations are adduced to demonstrate a potential retino-orbital perivascular outflow. Swelling of the optic nerve consequent upon demyelination may cause blockage of this retino-orbital perivascular channel. For the anatomical reasons stated, the oedema resulting from obstruction of this channel would be confined to the papilla.

A brief account is given of "senile papillitis", a syndrome resembling that which may follow cataract extraction.

Reasons are given for the concept of "neuronal retinopathy" to explain a wide range of bilateral amblyopias which, because of associated optic atrophy, have been attributed to optic neuritis.

I am grateful to Prof. Norman Ashton and Prof. Peter Daniel for examining the eyes and brain from the case of carbon dioxide poisoning. I also wish to thank Mr. D. P. Choyce and Dr. J. Colover for referring the Nigerian patient.

\section{REFERENCES}

Behr, C. Millen (1935). v. Graefes Arch. Ophthal., 134, 227.

BeHRMAN, S. (1962). Brit. J. Ophthal., 46, 554.

Berens, C., and PoSNer, A. (1933). Amer.J. Ophthal., 16, 19.

Breeman, V. L. van, and Clemente, C. D. (1955). J. biophys. biochem. Cytol., 1, 161.

BRUETSCH, W. L. (1947). Arch. Ophthal. (Chicago), 38, 735.

DANSEY-BrownING, G. C. (1958). Brit. J. Ophthal., 42, 394.

François, J., Verriest, G., NeETEnS, A., and RouCK, A. DE (1961). Bull. Soc. belge Ophtal., No. 129, 431. GIFFORD, H. (1886). Arch. Ophthal. (N.Y.), 15, 153.

GREENFIELD, J. G. (1958). "Neuropathology"', p. 134. Arnold, London.

HoYT, W. F. (1960). A.M.A. Arch. Ophthal., 64, 925.

HUBER, A. (1961). Ophthalmologica (Basel), 141, 290.

KATZ, J. L., VAlSAMIS, M. P., and JAMPEL, R. S. (1961). Amer. J. Ophthal., 52, 681.

KrǘCKMANN, E. (1917). Z.' Augenheilk., 37, 1.

KURZ, O. (1948). Ophthalmologica (Basel), 116, 281.

KWITTKEN, J., and BereST, H. D. (1958). Amer. J. Path., 34, 185.

LUCAS, D. R., and NewhOUSE, J. P. (1957). A.M.A. Arch. Ophthal., 58, 193.

MORGAN, O. GAYER (1952). Trans. ophthal. Soc. U.K., 73, 435.

NoELL, W. K. (1958). A.M.A. Arch. Ophthal., 60, 702.

PAPST, W. (1955). v. Graefes Arch. Ophthal., 157, 122.

Potts, A. M., Modrell, R. W., and KingsBury, G. (1960). Amer. J. Ophthal., 49, 900.

ReEse, A. B., and CARroll, F. D. (1958). Ibid., 45, 659.

RINTELEN, F. (1961). Ophthalmologica (Basel), 141, 283.

Schaltenbrand, G., and Bailey, P. (1928). J. Psychol. Neurol. (Lpz.), 35, 199.

SiNGH, S., and DASS, R. (1960a). Brit. J. Ophthal., 44, 280.

(1960b). Ibid., 44, 193.

SYMONDS, C. A. (1883). Brain, 5, 492 .

TANSLEY, K. (1956). Brit. J. Ophthal., 40, 178.

WeIZENBLATT, S. (1959). Amer.J. Ophthal., 47, 77.

WoLfF, E. (1951). "A Pathology of the Eye", 3rd ed., p. 307. Lewis, London.

WolfF, H. G. (1948). "Headache", p. 435. Oxford University Press, New York.

Wolter, J. R. (1957). Amer. J. Ophthal., 44, no. 4, pt 2, p. 48.

WOODWORTH, J. A., BECKETT, R. S., and NETSKY, M. G. (1959). Arch. intern. Med., 104, 594.

Woollam, D. H. M., and Millen, J. W. (1954). Biol. Rev., $29,251$. 